\title{
Evaluation of Sensitivity and Positive Predictive Values of Cytopathologic Diagnosis of Solid Masses in Dogs
}

\author{
Richard M. Kabuusu*, Tracy Volger, Sachin Kumthekar, Keshaw Tiwari, Alfred Chikweto, \\ Muhammad I. Bhaiyat, Claude De Allie, Ruth Alexander, Carla Richards, \\ Ravindra N. Sharma \\ Department of Pathobiology, School of Veterinary Medicine, St. George's University, St. George's, West Indies \\ Email: *rkabuusu@sgu.edu
}

Received 8 April 2016; accepted 20 June 2016; published 23 June 2016

Copyright (C) 2016 by authors and Scientific Research Publishing Inc.

This work is licensed under the Creative Commons Attribution International License (CC BY).

http://creativecommons.org/licenses/by/4.0/

(c) (i) Open Access

\section{Abstract}

In this retrospective study, a total of $\mathbf{2 7 5}$ solid masses were examined for cytopathologic diagnosis. Twenty four percent $(67 / 275)$ of these cytologic samples were followed by surgical biopsy and histopathologic diagnosis, allowing for comparisons. On average, the cutaneous and subcutaneous solid masses were recognized when the dogs were aged between 6 and 9 years old. The origins of the solid masses included connective tissue tumors $37.1 \%(23 / 62)$, epithelial tissue tumors $33.9 \%$ $(21 / 62)$, round cell tumors $19.4 \%(12 / 62)$, masses of inflammatory lesions $4.8 \%(3 / 62)$ and lesions due to other causes $4.8 \%(3 / 62)$. The sensitivity and positive predictive value (PPV) of cytopathology in the diagnosis of solid masses were $93 \%(62 / 67)$ and $97 \%(62 / 64)$, respectively. Generally, neo-plasms were over diagnosed by cytopathology as was indicated by the positive predictive value. Both the sensitivity and the PPV of cytopathology comparative to histopathology in the diagnosis of inflammatory processes were $100 \%(3 / 3)$. The inflammatory lesions were eventually confirmed as necrotizing myositis, necro-suppurative cystitis and endocrine inflammatory dermatopathy based on histopathology. Less than $8 \%(5 / 67)$ of samples were incorrectly diagnosed by cytology. The study showed high accuracy between cytological and histopathological examination of solid masses in dogs, and thus a reliable diagnostic tool in patient care.

\section{Keywords}

Cytopathology, Histopathology, Dog, Sensitivity, Positive Predictive Value, Solid Masses

\footnotetext{
${ }^{*}$ Corresponding author.
}

How to cite this paper: Kabuusu, R.M., et al. (2016) Evaluation of Sensitivity and Positive Predictive Values of Cytopathologic Diagnosis of Solid Masses in Dogs. Open Journal of Veterinary Medicine, 6, 95-98. 


\section{Introduction}

Diagnostic cytology involves the examination of cells which have either naturally exfoliated or have been artificially removed from a body cavity or a tissue mass [1]. The cytological interpretation is often valuable in defining a diagnosis, a disease process, a prognosis, directing therapy and determining any further diagnostic procedures. Some of the advantages of cytopathology over histopathology include the use of minimal equipment and reagents, and a rapid turnaround time. Cytopathology is minimally invasive thus, causing minimal stress levels to the patient [2] [3]. However, cytopathology has been mainly employed as a rapid screening tool, because its interpretation is based upon the distinct characteristics of individual cell populations that lack the source tissue architecture. On the other hand, histopathology usually provides more definitive diagnoses because it allows the study of both the structure and form of a tissue. This difference in the type of diagnostic specimens necessitates comparisons between cytopathologic and histopathologic diagnostic outcomes. A number of studies have been performed to evaluate the diagnostic accuracy of cytopathology compared to histopathology but relatively fewer studies have actually compared the diagnostic properties of cytopathology [4]-[9]. The purpose of this study is to provide further evidence that cytopathology of solid masses provides a powerful and reliable diagnostic tool in clinical and diagnostic settings. Other aims of the study include establishing the mean age of the dogs at the time of diagnosis, as well as to identify the anatomical location that is associated with the solid masses.

\section{Materials and Methods}

Approval from the Institutional Animal Care and Use Committee was not sought because this was a retrospective study. Samples of 275 solid masses in dogs submitted to the diagnostic laboratory within the School of Veterinary Medicine at St. George's University between 2008 through 2012 were included in the study. Evaluation was performed on solid masses either by Fine Needle Aspirations (FNA) or impression smear for cytological examination. The smears for cytopathological diagnosis were air dried, fixed with methanol and stained with diff-quik stain. Biopsy specimens from solid masses for histopathological diagnosis were fixed in $10 \%$ buffered formalin, processed routinely, embedded in paraffin, sectioned at $5 \mu \mathrm{m}$ and stained with hematoxylin and eosin (H \& E). The results of cytology and histology on 67 specimens were compared.

A diagnosis of a lesion was based on at least three pathologists working together or individually. Using histopathology as the gold standard, a cytopatholgical lesion was characterized as a true positive (TP) if the cytological diagnosis correlated with the histopathological diagnosis, or as a false positive (FP) if a cytological diagnosis was positive, but a negative histopathological diagnosis was obtained, and as a false negative (FN) when a positive histopathology diagnosis was made but no diagnosis was made cytologically. These were entered into $2 \times 2$ tables, and the sensitivity and positive predictive values of cytopathology compared to histopathology were calculated.

\section{Results}

Between 2008 and 2012, the incidence of solid masses was 1.4 per 1000 dogs per year resulting from 275 dogs with solid masses among 3813 dogs examined. There were 36 female dogs and 31 male dogs that were included in the study. It was not possible to calculate the specificity and negative predictive values (NPV) because there were no true negative (TN) animals given that all the dogs included in the study presented with solid masses that were due to either neoplasms or inflammatory processes.

The dogs had an average age of 6 and 7 years when a diagnosis of mast cell tumors and melanoma, respectively was made. Dogs were older (9 years old) when a diagnosis of carcinoma was made compared to mesechymal tumors (7 years old). Mast cells tumors were frequently located in the inguinal region, abdomen and limbs; melanomas were frequently located on eyelid and interdigital spaces; transmissible venereal tumors were mostly genital with a few extra-genital masses and lymphomas were not associated with a specific anatomical location. Solid masses that were associated with carcinomas frequently occurred in the mammary glands, dorsal thorax and abdomen, stomach, neck ears and front and hind limbs, while tumors of mesenchymal origin were located on the prescapular region, abdomen, thorax, liver, spleen and mandibles.

Overall, the sensitivity and PPV of cytopathology in classifying solid masses as inflammatory or neoplastic were 93\% (62/67) and 97\% (62/64), respectively. Neopalams were over-diagnosed by more than 3\%. Specifically, round cell neoplasms, carcinomas and sarcomas had PPV of 92\% (12/13), 88\% (21/24) and 88\% (23/26) and sensitivity of $100 \%(12 / 12), 100 \%(21 / 21)$ and $100 \%$ (23/23), respectively. Less than $8 \%(5 / 67)$ of samples 
were incorrectly diagnosed by cytology. Histological examination of these five cases revealed lesions consistent with fat necrosis with fibroplasia, follicular cyst, and chronic dermatitis and dermal fibroma.

Both sensitivity and PPV of cytopathology and histopathology in the diagnosis of inflammatory processes were $100 \%$ (3/3). The inflammatory lesions were eventually confirmed as necrotizing myositis, necro-suppurative cystitis and endocrine inflammatory dermatopathy based on histopathology.

\section{Discussion}

Not all cytologic samples were followed by histopathology because some of the dog-owners declined biopsy and/or necropsy for financial reasons.

At the time of sampling for cytopathology and biopsy (or necropsy) for histopathology, the majority of specimens (91\%) examined in this study were located cutaneously or subcutaneously while only $9 \%(6 / 67)$ were present in internal organs including kidney, liver, lung, stomach, and spleen. Previous studies had established that cutaneous and subcutaneous tissues were the major locations for tumors in dogs [10]. Whereas mast cell tumors, melanomas and TVT showed preference for certain anatomical locations [11]-[13], most neoplasms originating from epithelial and mesechymal tissue were non-selective in the anatomical location.

The findings in this study established that cytopathology had high sensitivity and positive predictive values in the diagnosis of cutaneous and subcutaneous solid masses. Previous researchers reported high correlations between cytopathologic and histopathologic diagnosis of solid masses that ranged between $71.4 \%$ to $100 \%$ [5] [6] [14] [15]. The variation in these correlations was associated with sampling a different situate of the solid mass [9] [11], as well as sampling different tissues. The highest correlation had been reported for musculoskeletal lesions (100\%) followed by skin and subcutaneous lesions (91\%), gastrointestinal tract (76\%) and the lowest correlation for splenic lesions (38\%) [9] [11]. Over-diagnosis of neoplasms especially those of epithelial or mesenchymal origin illustrated the difficulty associated with their accurate interpretation and might be caused by dysplastic or hyperplastic changes. A major limitation of this study was that it was not possible to establish the age of the dog when the solid mass first occurred, thus the age of the dog when a diagnosis made was used instead.

Despite the modest sample size, our study showed that the sensitivity and positive predictive value of cytopathology in diagnosing of solid masses in dogs was high. Thus, cytopathology provided quick diagnostically-reliable results in clinical settings, but care must be taken not to over-diagnose.

\section{Acknowledgements}

The authors are grateful to the technical staff of the Veterinary Diagnostic Laboratory within the School of Veterinary Medicine at the St. George's University.

\section{Competing Interest}

The authors wish to declare that there is no competing interest.

\section{References}

[1] Menard, M., Fontanie, M. and Morin, M. (1986) Fine Needle Aspiration Biopsy of Malignant Tumors of Dogs and Cats: A Report of 102 Cases. Canadian Veterinary Journal, 27, 504-510.

[2] Lever, J.V., Trott, P.A. and Webb, A.J. (1985) Fine Needle Aspiration Cytology. Journal of clinical pathology, 38, 111. http://dx.doi.org/10.1136/jcp.38.1.1

[3] Kocjan, G., Nayagam, M. and Harris, M. (1990) Fine Needle Aspiration Cytology of Salivary Gland Lesions: Advantages and Pitfalls. Cytopathology, 1, 269-275. http://dx.doi.org/10.1111/j.1365-2303.1990.tb00360.x

[4] Stirtzinger, T. (1988) The Cytologic Diagnosis of Mesenchymal Tumors. Seminars in Veterinary Medicine and Surgery (Small Animal), 3, 157-166.

[5] Griffiths, G., Lumsden, J. and Vall, V. (2004) Fine Needle Aspiration Cytology and Histologic Correlation of Canine Tumors. Veterinary Clinical Pathology, 13, 13-17. http://dx.doi.org/10.1111/j.1939-165X.1984.tb00628.x

[6] Ghisleni, G., Roccabianca, P., Ceruti, R., Stefanello, D., Bertazzolo, W., Bonfanti, U. and Caniatti, M. (2006) Correlation between fine-Needle Aspiration Cytology and Histopathology in the Evaluation of Cutaneous and Subcutaneous Masses from Dogs and Cats. Veterinary Clinical Pathology, 35, 24-30.

http://dx.doi.org/10.1111/j.1939-165X.2006.tb00084.x 
[7] Simeonov, R.S. (2012) The Accuracy of Fine-Needle Aspiration Cytology in the Diagnosis of Canine Skin and Subcutaneous Masses. Comparative Clinical Pathology, 21, 143-147. http://dx.doi.org/10.1007/s00580-010-1075-5

[8] Sharkey, L.C., Dial, S.M. and Matz, M.E. (2007) Maximizing the Diagnostic Value of Cytology in Small Animal Practice. Veterinary Clinics of North America: Small Animal Practice, 37, 351-372. http://dx.doi.org/10.1016/j.cvsm.2006.11.004

[9] Cohen, M., Bohling, M.W., Wright, J.C., Welles, E.A. and Spano, J.S. (2003) Evaluation of Sensitivity and Specificity of Cytologic Examination: 269 Cases (1999-2000). Journal of the American Veterinary Medical Association, 222, 964967. http://dx.doi.org/10.2460/javma.2003.222.964

[10] Dobson, J.M. and Gorman, N.T. (1988) A Clinical Approach to the Management of Skin Tumours in the Dog and Cat. In Practice, 10, 55-68. http://dx.doi.org/10.1136/inpract.10.2.55

[11] Al-Sarraf, R., Mauldin, G.N., Patnaik, A.K. and Meleo, K.A. (1996) A Prospective Study of Radiation Therapy for the Treatment of Grade 2 Mast Cell Tumors in 32 Dogs. Journal of Veterinary Internal Medicine, 10, 376-378. http://dx.doi.org/10.1111/j.1939-1676.1996.tb02084.x

[12] Sfiligoi, G., Rassnick, K.M., Scarlett, J.M., Northrup, N.C. and Gieger, T.L. (2005) Outcome of Dogs with Mast Cell Tumors in the Inguinal or Perineal Region versus Other Cutaneous Locations: 124 Cases (1990-2001). Journal of the American Veterinary Medical Association, 226, 1368-1374. http://dx.doi.org/10.2460/javma.2005.226.1368

[13] Spangler, W.L. and Kass, P.H. (2006) The Histologic and Epidemiologic Bases for Prognostic Considerations in Canine Melanocytic Neoplasia. Veterinary Pathology Online, 43, 136-149. http://dx.doi.org/10.1354/vp.43-2-136

[14] Vos, J.H., van den Ingh, T.S.G.A.M. and Van Mil, F.N. (1989) Non-Exfoliative Canine Cytology: The Value of Fine Needle Aspiration and Scraping Cytology. Veterinary Quarterly, 11, 222-231. http://dx.doi.org/10.1080/01652176.1989.9694228

[15] Eich, C.S., Whitehair, J.G., Moroff, S.D. and Heeb, L.A. (2000) The Accuracy of Intraoperative Cytopathological Diagnosis Compared with Conventional Histopathological Diagnosis. Journal of the American Animal Hospital Association, 36, 16-18. http://dx.doi.org/10.5326/15473317-36-1-16 